\title{
STRUCTURAL STATE OF NANOCOMPOSITES BASED ON HIGHLY EXFOLIATED GRAPHITE AND 3D-TRANSITION METALS
}

\author{
E. G. Gerasimov ${ }^{1,2}$, P. B. Terentiev ${ }^{1,2}$, N. N. Shchegoleva ${ }^{1}$, N. V. Mushnikov ${ }^{1,2}$, B. Campbell $^{3}$,
} A. N. Pirogov ${ }^{1,2^{*}}$, Yu. N. Scryabin ${ }^{1}$, A. E. Teplykh ${ }^{1}$, S. G. Bogdanov ${ }^{1}$, V. E. Fedorov ${ }^{4}$, N. G. Naumov ${ }^{4}$, A. P. Vokhmyanin ${ }^{1}$, V. V. Bobrovsky ${ }^{1}$

${ }^{I}$ M.N. Miheev Institute of Metal Physics, Ural Branch of the Russian Academy of Sciences, 18 S. Kovalevskoy St., Ekaterinburg, Russian Federation,

${ }^{2}$ Institute of Natural Sciences and Mathematics, B. N. Yeltsin Ural Federal University, 19 Mira St., Ekaterinburg, Russian Federation

${ }^{3}$ Brigham Young University, Provo, Utah, USA

${ }^{4}$ Nikolaev Institute of Inorganic Chemistry, Siberian Branch of the Russian Academy of Sciences, Novosibirsk, Russian Federation

\author{
*Corresponding author. E-mail: pirogov@imp.uran.ru \\ Address for correspondence: 18, ul. S. Kovalevskoy, 620137, Ekaterinburg, Russian Federation \\ Tel.: 895305076 54; fax: 83433740003
}

The structural state and magnetic properties of nanocomposites formed by exfoliated graphite and 3d-transition metal (Co or $\mathrm{Ni}$ ) particles have been studied. The exfoliated graphite was synthesized by thermal decomposition of the intercalated graphite $\mathrm{C}_{2} \mathrm{Fx}\left(\mathrm{BrF}_{3}\right)$. The thus synthesized exfoliated graphite is multilayer graphene. The salt $\mathrm{CoCl}_{2} \cdot 6 \mathrm{H}_{2} \mathrm{O}$ (or $\mathrm{NiCl}_{2} \cdot 6 \mathrm{H}_{2} \mathrm{O}$ ) was added to the graphene, and the mixture was agitated by a stirrer for half an hour and then heated in a hydrogen flow. The microstructure of the nanocomposites represents separated practically spherical inclusions of $\mathrm{Co}$ (or $\mathrm{Ni}$ ) nanoparticles into a multilayer graphene matrix. The $\mathrm{X}$-ray diffraction patterns of the nanocomposites with Co particles testify to their two-phase state at $293 \mathrm{~K}$ : they crystallize in low-temperature hexagonal and high-temperature cubic phases. At $78 \mathrm{~K}$ and $293 \mathrm{~K}$, the magnetization curves of the nanocomposites, which are measured in pulsed magnetic fields of up to $100 \mathrm{kOe}$, look typically of ferromagnets.

Keywords: multilayer graphene, 3d-transition metals, nanocomposites.

\section{Acknowledgments}

This research was carried out within the SA of FASO of Russia (themes "Magnet" No. 0120146332 and "Flux" No. 01201463334).

DOI: $10.17804 / 2410-9908.2017 .6 .120-127$

\section{References}

1. Zhu J., Wei S., Zhang L., Mao Y., Ryu J., Karki A.B., Young D.P., Guo Z. Polyanilinetungsten oxide meta-composites with tunable electronic properties. J. Mater. Chem., 2011, vol. 21, pp. 342-348. DOI: 10.1039/c0jm02090g

2. Kamat P.V. Graphene_based nanoarchitectures. Anchoring semiconductor and metal nanoparticles on a two-dimensional carbon support. J. Phys. Chem. Letters, 2010, vol. 1, pp. 520-527

3. Chandra V., Park J., Chun Y., Lee J.W., Hwang I.C., Kim K.S. Water-Dispersible Magnetite-Reduced Graphene Oxide Composites for Arsenic Removal. ACS Nano., 2010, vol. 4, pp. 39793986. DOI: $10.1021 / \mathrm{nn} 1008897$

4. Tombros N., Jozsa C., Popinciuc M., Jonkman H.T., Van Wees B.J. Electronic spin transport and spin procession in single graphene layers at room temperature. Nature, 2007, vol. 448, pp. 571-574. DOI:10.1038/nature06037

5. Teplykh A.E., Bogdanov S.G., Gerasimov E.G., Terentev P.B., Korolev A.V., Fedorov V.E., Makotchenko V.G., Naumov N.G., Kampbell B.D., Pirogov A.N. Structure state and magnetic 
hittp://dream-journal.op"n

propeties of multilayer graphene/Fe composites. Phys. Met. Met., 2016, vol. 117, no. 2, pp. $22-29$. DOI: $10.7868 / \mathrm{S} 001532301602011 \mathrm{X}$

6. Makotchenko V.G., Grayfer E.D., Nazarov A.S., Kim S.-J., Fedorov V.E. The synthesis and properties of highly exfoliated graphites from fluorinated graphite intercalation compounds. Carbon, 2011, vol. 49, pp. 3233-3241. DOI:10.1016/j.carbon.2011.03.049.7

7. Teplykh A.E., Bogdanov S.G., Dorofeev Yu.A., Pirogov A.N., Skryabin Yu.N., Makotchenko V.G., Nazarov A.S., Fedorov V.E. Structural state of expanded graphite prepared from intercalation compounds. Crystallography Reports, 2006, vol. 51, no. Suppl. 1, pp. S62- S66. DOI: $10.1134 / \mathrm{S} 1063774506070108$ 
Подана в журнал: 04.12.2017

УДК 669.784'1:539.27

DOI: $10.17804 / 2410-9908.2017 .6 .120-127$

\title{
СТРУКТУРНОЕ СОСТОЯНИЕ НАНОКОМПОЗИТОВ НА ОСНОВЕ ВЫСОКОРАСЩЕПЛЕННОГО ГРАФИТА И Зd-ПЕРЕХОДНЫХ МЕТАЛЛОВ
}

\author{
Е. Г. Герасимов ${ }^{1,2}$, П. Б. Терентьев ${ }^{1,2}$, Н. Н. Щеголева ${ }^{1}$, Н. В. Мушников ${ }^{1,2}$, \\ Б. Кэмпбелл ${ }^{3}$, А. Н. Пирогов ${ }^{1,2 *}$, Ю. Н. Скрябин ${ }^{1}$, А. Е. Теплых ${ }^{1}$, С. Г. Богданов ${ }^{1}$, \\ В. Е. Федоров ${ }^{4}$, Н. Г. Наумов ${ }^{4}$, А. П. Вохмянин ${ }^{1}$, В. И. Бобровский ${ }^{1}$ \\ ${ }^{1}$ ФБУН Институт физики металлов имени М.Н. Михеева \\ Уральского отделения Российской академии наук, ул. С. Ковалевской, 18, Екатеринбург, Российская Федерация \\ ${ }^{2}$ Институт естественных наук и математики, Уральский федеральный университет, \\ ул. Мира, 19, Екатеринбург, Российская Федерация \\ ${ }^{3}$ Бригхам Янг университет, Прово, Юта, США \\ ${ }^{4}$ ФГБУН Институт неорганической химии им. А.В. Николаева \\ Сибирского отделения Российской академии наук, Новосибирск, Российская Федерация \\ *Ответственный автор. Электронная почта: pirogov@imp.uran.ru \\ Адрес для переписки: 620137, Екатеринбург, ул. С. Ковалевской, 18, Российская Федерация \\ Тел.: 895305076 54; факс: 83433740003
}

Исследовано структурное состояние и магнитные свойства нанокомпозитов, сформи-

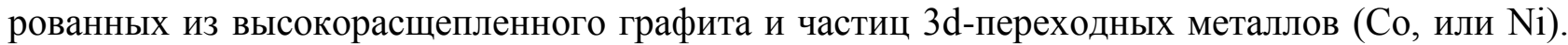
Высокорасщепленный графит был синтезирован термическим разложением интеркалированного соединения окисленного графита $\mathrm{C}_{2} \mathrm{Fx}\left(\mathrm{BrF}_{3}\right)$. Синтезированный таким способом высокорасщепленный графит представляет собой мультислойный графен. Полученный мультислойной графен смешивался с солью $\mathrm{CoCl}_{2} \cdot 6 \mathrm{H}_{2} \mathrm{O}$ (или $\mathrm{NiCl}_{2} \cdot 6 \mathrm{H}_{2} \mathrm{O}$ ), и смесь в течение получаса нагревалась в потоке водорода. Микроструктура нанокомпозитов представляет собой изолированные практически сферические включения наночастиц Со и $\mathrm{Ni}$ в матрицу мультислойного графена. Рентгенограммы нанокомпозитов с кобальтом свидетельствуют, что при 293 К Со частицы находятся в двухфазном состоянии: в низкотемпературной гексагональной и высокотемпературной кубической фазе. Кривые намагничивания нанокомпозитов, измеренные в импульсных магнитных полях с напряженностью до 100 кЭ при температурах 78 и 293 К, имеют вид, типичный для ферромагнетика.

Ключевые слова: мультислоевой графен, 3d-переходный металл, нанокомпозит.

\section{1. Введение}

В литературе наблюдается повышенное внимание к исследованию различных композитов, что связано с поиском новых функциональных материалов с уникальными физическими свойствами, которые могут найти применение в новых устройствах и конструкциях [1]. Хотя требования к многофункциональным материалам постоянно возрастают, экспериментальные методики и технологии также совершенствуются, и это дает надежды на открытие новых видов композиционных материалов. В этом отношении открытие графена и основанных на графене нанокомпозитов представляет важное направление синтеза новых материалов с необычными свойствами. Большинство графеновых нанокомпозитов, исследованных к настоящему времени, содержали в качестве допирующего элемента благородные металлы $(\mathrm{Pt}, \mathrm{Pd}, \mathrm{Ru}, \mathrm{Rh}, \mathrm{Ir}, \mathrm{Au}, \mathrm{Ag})[2,3]$. Графеновые слои, декорированные магнитными частицами, такими как $\mathrm{Fe}, \mathrm{Co}, \mathrm{Ni}$, были изучены с точки зрения применения их в батареях, установках для сорбции органических загрязнений и т. д. Было обнаружено, что граничный слой в ком-

Gerasimov E.G. et al. / Structural state of nanocomposites based on highly exfoliated graphite

and $3 \mathrm{~d}$-transition metals 
позитах, содержащих ферромагнитный металл, проявляет свойства, которые могут быть использованы в спиновых клапанах [4]. Перспективным направлением представляется и применение магнитных композитов в медицине: магнитные нанокомпозиты по сравнению с другими носителями лекарства отличаются возможностью бесконтактного управления ими в организме человека с помощью внешнего магнитного поля.

Цель данной работы заключалась в получении и исследовании структуры и магнитных свойств композитов на основе высокорасщепленного графита и наночастиц 3d-переходных металлов (Со и $\mathrm{Ni})$.

\section{2. Материал и методика эксперимента}

Образцы нанокомпозитов на основе высокорасщепленного графита и наночастиц 3d-переходных металлов с различной концентрацией $\mathrm{Ni}$ и Со были получены следующим образом. Высокорасщепленный графит был синтезирован термическим разложением при $800{ }^{\circ} \mathrm{C}$ интеркалированного соединения окисленного графита $\mathrm{C}_{2} \mathrm{~F} \times\left(\mathrm{BrF}_{3}\right)$ [5]. Достоинством этого метода является очень низкое содержание кислорода в полученном материале. Синтезированный таким способом высокорасщепленный графит представляет собой мультислойный графен, содержащий небольшое число слоев (в среднем не больше 10) с увеличенным на $\sim 4 \%$ расстоянием между ними. Этот материал обладает удельной поверхностью, достигающей $300 \mathrm{~m}^{2} / \Gamma$ и является высокоэффективным сорбентом для органических загрязнений. Исследование его структурного состояния проводилось нами ранее в работах $[6,7]$.

К полученному мультислойному графену массой 100 мг добавляли расчетное количество солей $\mathrm{CoCl}_{2} \cdot 6 \mathrm{H}_{2} \mathrm{O}\left(\mathrm{NiCl}_{2} \cdot 6 \mathrm{H}_{2} \mathrm{O}\right)$, растворенных в этиловом спирте. Эту смесь перемешивали в течение получаса, при этом растворенные соли адсорбировались на поверхности мультислойного графена. Полученную массу сушили на воздухе в течение 8 ч и прессовали в таблетки диаметром 12 мм. Затем таблетки нагревали до $400{ }^{\circ} \mathrm{C}$ в потоке водорода в кварцевом реакторе и выдерживали в течение 2 ч. Синтезированный композит охлаждали в потоке водорода до комнатной температуры. В таком процессе соли кобальта (никеля) полностью восстанавливаются до металлического состояния. Были получены два образца с содержанием частиц кобальта 39 вес. \% (в дальнейшем как RG_1Co) и 29 вес. \% (RG_2Co) и два образца с концентрацией частиц никеля, 62 вес. \% и 34 вес. \% соответственно (RG_3Ni) и (RG_4Ni).

Образцы для измерений были размолоты в порошок с размером частиц около 20 микрон. Микроструктуру образцов исследовали с помощью электронного микроскопа JЕМ200. Рентгенограммы получены с помощью дифрактометра ДРОН-3 в Cr-K $\mathrm{K}_{\alpha}$ излучении. Магнитные измерения проводили в диапазоне температур 2-300 К, используя СКВИД-магнитометр Quantum Design MPMS5XL и магнитометр на основе импульсного соленоида в Центре коллективного пользования ИФМ УрО РАН.

\section{3. Результаты и их обсуждение}

Микроструктура исследованных в данной работе нанокомпозитов представляет собой изолированные практически сферические включения наночастиц Со и $\mathrm{Ni}$ в матрицу мультислойного графена (рис. $1 a$, б). Различие между образцами заключается только в количестве частиц в заданном объёме и в распределении по размерам частиц и их однородности. Размеры частиц никеля и кобальта варьируются от нескольких нанометров до двух сотен нанометров. На поверхности частиц 3d-переходных металлов образуется углеродное покрытие, препятствующее их окислению (рис. 1 в). Графеновая матрица имеет складчатую структуру. Ширина складок неодинаковая и составляет 3-40 нм, их толщина также различна, изменяется в среднем от двух до десяти слоев (различный контраст на изображении). На участках матрицы без складок и внутри складок весьма заметен неоднородный контраст, который можно связывать с различной ориентированностью разных фрагментов пленки, размер этих

Gerasimov E.G. et al. / Structural state of nanocomposites based on highly exfoliated graphite

and 3d-transition metals 


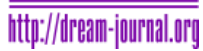

неоднородностей около 2,5-5 нм. Все эти морфологические особенности углеродной матрицы проявляются на полученных с этой матрицы кольцевых электронограммах.

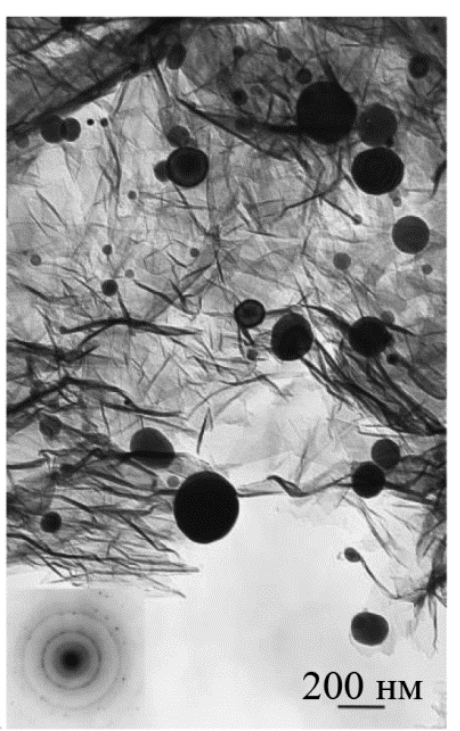

$a$

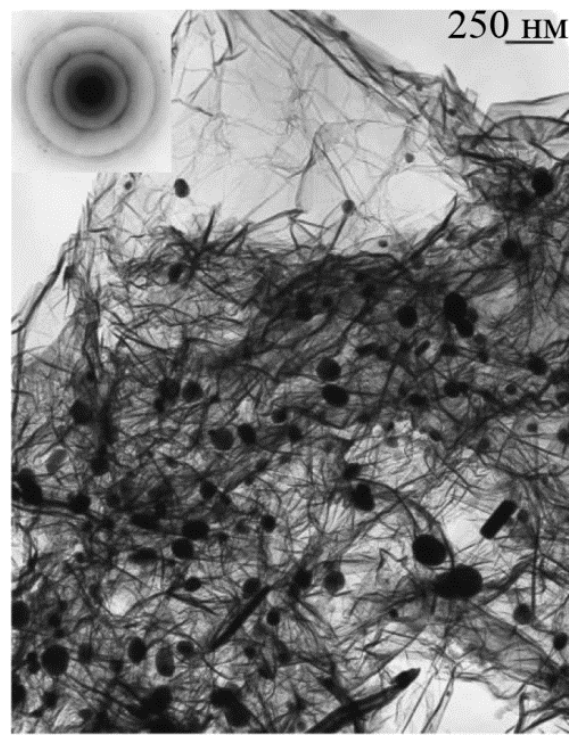

$\sigma$

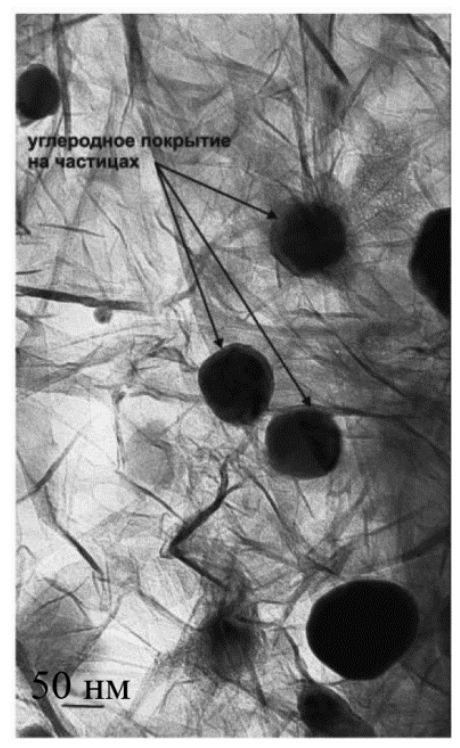

B

Рис. 1. Микроструктура композитов RG_3Ni $(a)$ и RG_1Co $(\sigma$, в $)$

На рис. 2 и 3 представлены рентгенограммы четырех исследованных нами образцов нанокомпозитов. Рефлексы с большой шириной обусловлены рассеянием на графене, а относительно узкие отражения связаны с рассеянием на частицах $3 \mathrm{~d}$-переходных металлов. Из данных о полуширине рефлексов мы оценили размеры частиц графена (около 4-5 нм)

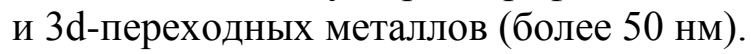

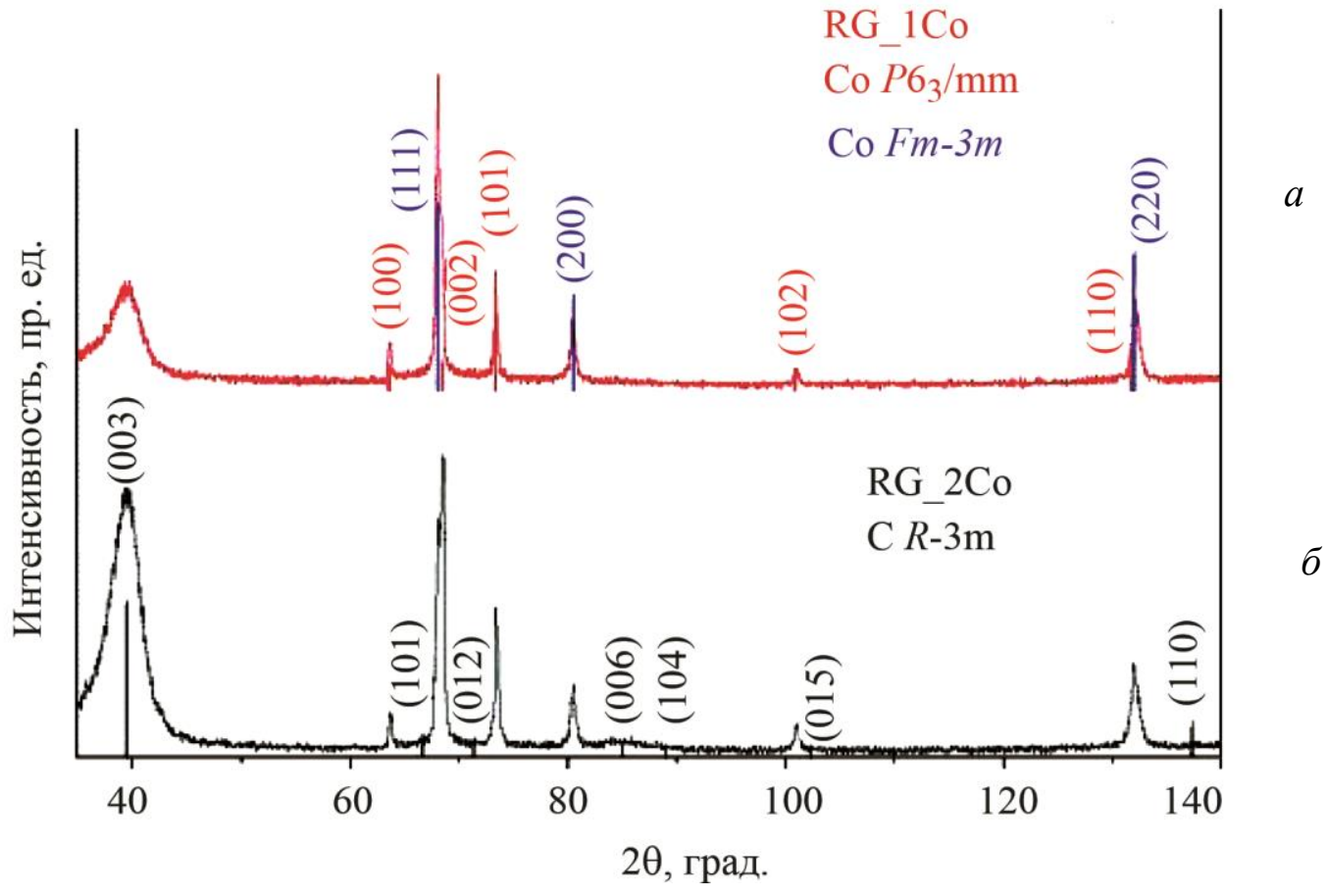

Рис. 2. Рентгенограммы композитов RG1_Co (a) и RG2_Co (б)

Gerasimov E.G. et al. / Structural state of nanocomposites based on highly exfoliated graphite and 3d-transition metals 


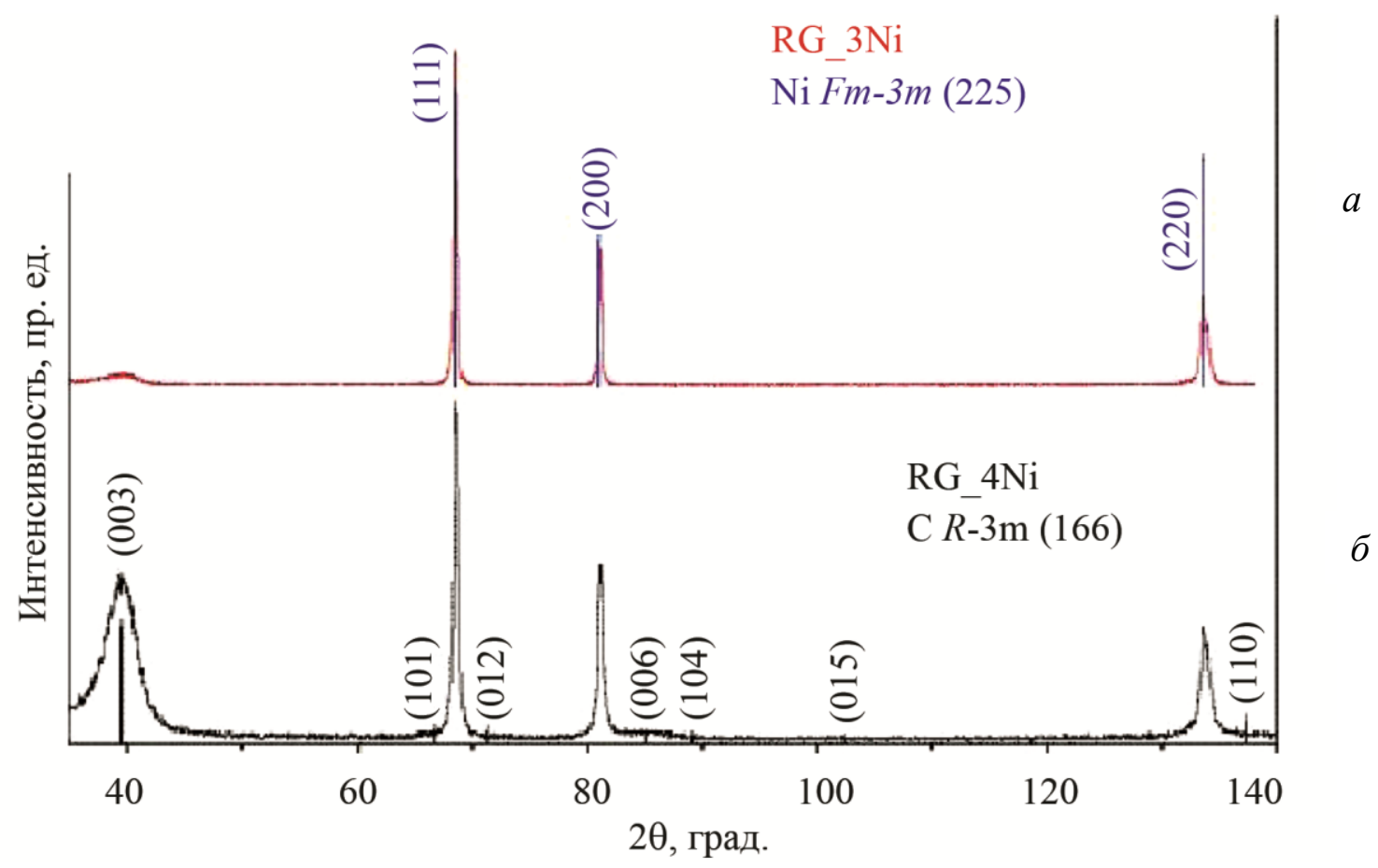

Рис. 3. Рентгенограммы композитов RG_3Ni (a) и RG_4Ni (б)

Мы обнаружили, что наличие контакта между мультислойным графеном и частицами кобальта приводит к значительному понижению температуры структурного перехода гексагональная плотноупакованная - кубическая гранецентрированная фаза кобальта. Как известно, этот переход в массивном кобальте происходит при 700 К. На рис. 2, вверху (RG_1Co, Co P6 63 mmc (194) Co Fm-3m (225) видно, что при комнатной температуре существуют рефлексы, принадлежащие как гексагональной $(h k l=(100),(002),(102),(110))$, так и кубической $(h k l=(111),(200),(220))$ фазам. Следовательно, данные композиты не есть простая механическая смесь частиц мультислойного графена и кобальта, взаимодействующие подсистемы. Одновременное наличие в композитах двух фаз кобальта, может обусловливать новые физико-химические свойства этих композитов, которые могут найти новые применения.

Кривые намагничивания нанокомпозитов, измеренные в импульсных магнитных полях с напряженностью до 100 кЭ при температурах 78 и 293 К, имеют вид, типичный для ферромагнетика (рис. 4). Насыщение на кривых намагничивания достигается уже при небольших значениях напряженности магнитного поля, а численные значения намагниченности в насыщении соответствуют весовому содержанию 3d-переходного металла в композите и согласуются с весовым содержанием 3d-переходного металла, определенного химическим способом. Ферромагнитное поведение нанокомпозитов обусловлено большими размерами частиц 3d-переходного металла, превышающими критический диаметр суперпарамагнетизма $D_{\mathrm{s}}$, который можно оценить как:

$$
D_{\mathrm{s}}=\left(150 k_{\mathrm{B}} T / \pi|K|\right),
$$

где $k_{\mathrm{B}}$ - постоянная Больцмана; $T$ - температура; $K$ - константа магнитной анизотропии. Для кобальта константа магнитной анизотропии при 4,2 К и $293 \mathrm{~K}$ равна $7 \cdot 10^{6}$ Эрг/см ${ }^{3}$

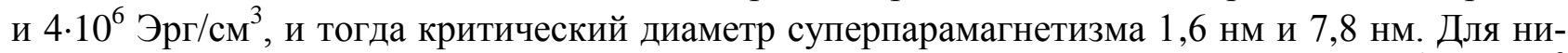
келя первая константа магнитной анизотропии при 4,2 К и 293 К равна $-1,2 \cdot 10^{6}$ Эрг/см

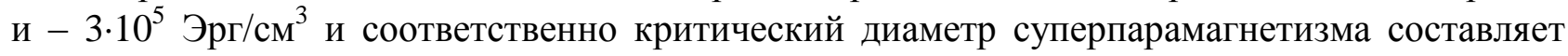
2,8 нм и 18 нм. На кривых размагничивания нанокомпозитов практически отсутствует магнитный гистерезис, что может свидетельствовать о том, что частицы кобальта и никеля находятся в многодоменном магнитном состоянии.

Gerasimov E.G. et al. / Structural state of nanocomposites based on highly exfoliated graphite and 3d-transition metals 


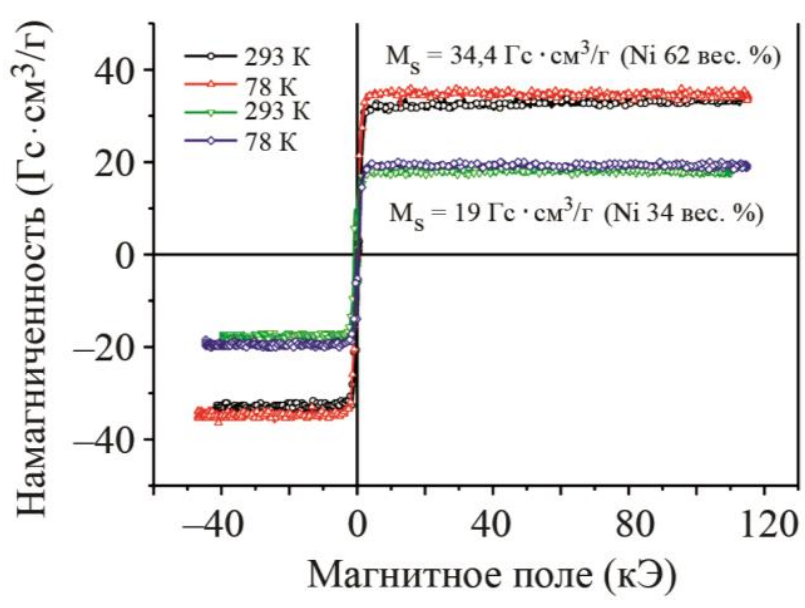

$a$

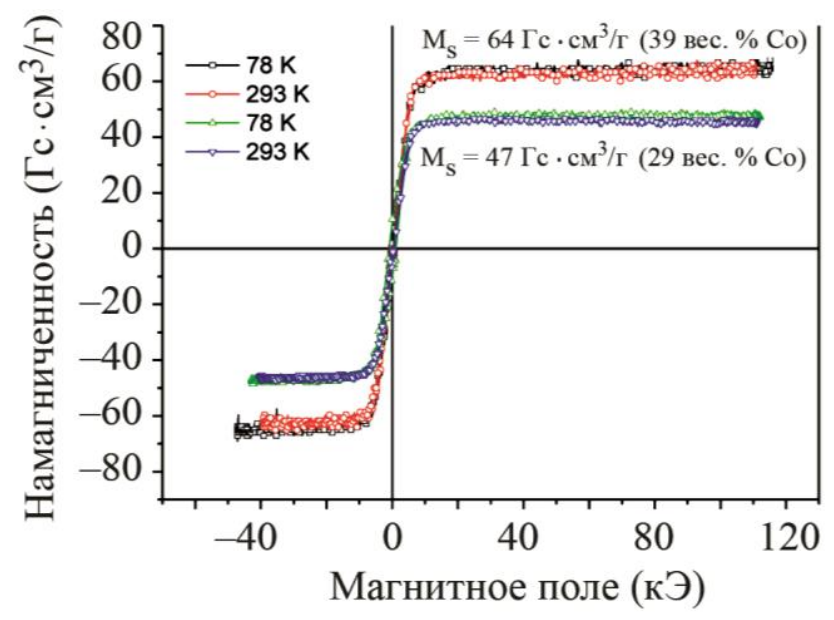

$\sigma$

Рис. 4. Петли гистерезиса композитов RG_3Ni и RG_4Ni (a); RG_1Co и RG_2Co (б), измеренные при температурах 78 и $293 \mathrm{~K}$

\section{4. Выводы}

В работе получены нанокомпозиты на основе мультислойного графена и наночастиц 3d-переходных металлов: Со с концентрацией 29 вес. \% и 39 вес. \% и Ni с концентрацией 34 вес. \% и 62 вес. \%.

Проведенные исследования структурного состояния и магнитных свойств нанокомпозитов показали, что в полученных таким способом образцах средние размеры толщины слоев мультислойного графена составляют около 4 нм, а размеры частиц Со и Ni составляют более 50 нм. Магнитные свойства нанокомпозитов определяются магнитными свойствами частиц Со и $\mathrm{Ni}$.

Обнаружено, что между мультислойным графеном и частицами кобальта существует взаимодействие, которое приводит к понижению температуры перехода гексагональная плотноупакованная - кубическая гранецентрированная фазы кобальта, примерно, на 400 К.

\section{Благодарность}

Работа выполнена в рамках государственных заданий ФАНО России (темы «Магнит», № 01201463328 и «Поток», № 01201463334).

\section{Список литературы}

1. Polyaniline-tungsten oxide meta-composites with tunable electronic properties / J. Zhu, S. Wei, L. Zhang, Y. Mao, J. Ryu, A. B. Karki, D. P. Young, Z. Guo // J. Mater. Chem. - 2011. Vol. 21. - P. 342-348. - DOI: 10.1039/c0jm02090g

2. Kamat P. V. Graphene_based nanoarchitectures. Anchoring semiconductor and metal nanoparticles on a two-dimensional carbon support // J. Phys. Chem. Letters. - 2010. - Vol. 1. - P. 520-527.

3. Water-Dispersible Magnetite-Reduced Graphene Oxide Composites for Arsenic Removal / V. Chandra, J. Park, Y. Chun, J. W. Lee, I. C. Hwang, K. S. Kim // ACS Nano. - 2010. - Vol. 4. P. 3979-3986. - DOI: 10.1021/nn1008897

4. Electronic spin transport and spin procession in single graphene layers at room temperature / N. Tombros, C. Jozsa, M. Popinciuc, H. T. Jonkman, B. J. Van Wees // Nature. - 2007. - Vol. 448. P. 571-574. - DOI: 10.1038/nature06037

5. Structure state and magnetic propeties of multilayer graphene/Fe composites / A. E. Teplykh, S. G. Bogdanov, E. G. Gerasimov, P. B. Terentev, A. V. Korolev, V. E. Fedorov, V. G. Makotchenko, 
N. G. Naumov, B. D. Kampbell, A. N. Pirogov // Phys. Met. Met. - 2016 - Vol. 117, no. 2. P. 22-29. - DOI: 10.7868/S001532301602011X

6. The synthesis and properties of highly exfoliated graphites from fluorinated graphite intercalation compounds / V. G. Makotchenko, E. D. Grayfer, A. S. Nazarov, S.-J. Kim, V. E. Fedorov // Carbon. - 2011. - Vol. 49. - P. 3233-3241. - DOI: 10.1016/j.carbon.2011.03.049.

7. Structural state of expanded graphite prepared from intercalation compounds / A. E. Teplykh, S. G. Bogdanov, Yu. A. Dorofeev, A. N. Pirogov, Yu. N. Skryabin, V. G. Makotchenko, A. S. Nazarov, V. E. Fedorov // Crystallography Reports. - 2006. - Vol. 51, Suppl. 1. - P. S62-S66. DOI: $10.1134 / \mathrm{S} 1063774506070108$ 